\title{
Model-Based Controls Development and Implementation for a Hydroelectric Power System
}

\author{
Anh Nguyen ${ }^{1}$ John Batteh ${ }^{2}$ \\ ${ }^{1}$ Modelon Inc., USA, \{anh.nguyen, john. batteh $\}$ modelon.com
}

\begin{abstract}
This paper describes the model-based control system development for a hydroelectric power plant to ensure water level control and mitigate spillage risk. The modeling of both the flume system and prototype controls is described. The integrated model is run over a suite of tests to verify the calibration of the control strategy. Results from the plant commissioning are compared with the virtual tests. The model proved capable of accurate predictions of the waterway dynamics, and the model-based calibration was successfully verified on the actual plant.
\end{abstract}

Keywords: hydro power, control systems; hydraulics

\section{Introduction}

Hydroelectric power plants can experience long lifecycles with plants often operating for decades. With years of historical data, it is not uncommon for plants to operate based on manual operator control. In an effort to improve power dispatch and uptime, optimize revenue, extend plant life, and improve reliability and safety, modern control solutions can be deployed and retrofit to existing plants at significant benefit. Modelbased controls development is a critical element for any plant control modernization effort.

Hydro Power Library (Modelon AB, 2018) provides a framework for modeling and simulation of hydro power plant operation and control. The library provides a complete environment for modeling the plant system including the hydraulics, waterway dynamics, plant turbine and electrical, and associated controls including both dynamic and steady state operation. A model of the Sundsbarm hydro power plant in Seljorn, Norway was built using a previous version of the library to simulate and identify the reasons for power production variation at the plant including the development of a linearized model and a model predictive control (MPC) approach to optimize plant operation (Winkler et al, 2011). The library has been used to simulate a number of on and off-design operating conditions for the Fossárvirkjun power station in northern Iceland (Magnúsdóttir and Winkler, 2017) including a detailed electrical system modeled with Electric Power Library.

This paper describes the model-based development of a control strategy for a hydro power plant to ensure appropriate water level control for environmental impact due to spillage risk. Due to US security concerns for critical power infrastructure, the plant must remain anonymous with some sensitive data obscured. The paper will provide an overview of the waterway system model including model calibration. The control strategy is prototyped and integrated with the waterway system model. A virtual test suite is executed for model-based calibration and verification of the control strategy. Results from the model are then compared with data obtained from commissioning and testing on the actual plant. The model-based approach proved capable of predicting the waterway dynamics and for model-based calibration and verification of the control strategy.

\section{Flume System Modeling}

This section describes the physical model of the flume system. The model is assembled using Hydro Power Library (Modelon AB, 2018) along with some custom components. The full hydraulic system model is introduced and then individual components are described in more detail along with the calibration performed based on available data from the waterway prior to commissioning.

\subsection{Full System Model}

Figure 1 shows the full hydraulic system model starting from the intake of the flume system and ending at the turbine inlet. The model includes the following components:

- Intake gate with control

- Upper flume system with rectangular geometry

- Lower flume system with trapezoidal geometry

- Flume at spillway for increased resolution at critical area for spillage

- Basin and forebay reservoirs

- Penstock with valve control

- Visualizers for flow rate, water elevation, and volume at various locations in the flume system

- Visualizer component to show invert elevation, water elevation, and max elevation throughout the flume system 


\begin{tabular}{|c|c|c|c|c|c|c|c|c|c|c|c|}
\hline \multicolumn{3}{|c|}{ UpperFlume } & & \multicolumn{2}{|c|}{ Basin } & \multicolumn{3}{|c|}{ LowerFlume } & & \multicolumn{2}{|c|}{ Forebay } \\
\hline \multirow{3}{*}{$Q$} & $\mathrm{~m} 3 / \mathrm{s}$ & $\mathrm{ft} 3 / \mathrm{s}$ & \multirow{3}{*}{ V } & $\mathrm{m}^{3}$ & $\mathrm{ft3}$ & \multirow{3}{*}{$Q$} & $\mathrm{n} 3 / \mathrm{s}$ & $\mathrm{ft} 3 / \mathrm{s}$ & \multirow{3}{*}{ V } & $\mathrm{m} 3$ & $\mathrm{ft} 3$ \\
\hline & 0.0 & 0.0 & & 00 & 0.0 & & 0.0 & 0.0 & & 0.0 & 0.0 \\
\hline & $\mathrm{m}$ & $\mathrm{ft}$ & & $\mathrm{m}$ & $\mathrm{ft}$ & & $\mathrm{m}$ & $\mathrm{ft}$ & & $\mathrm{m}$ & $\mathrm{ft}$ \\
\hline $\mathrm{H}[1]$ & 0.0 & 0.0 & $\mathrm{H}$ & 0 & 00 & \multirow{2}{*}{$\begin{array}{c}\mathrm{H}[1] \\
\mathrm{H}[\text { end] }\end{array}$} & 0.0 & 0.0 & \multirow{2}{*}{$\begin{array}{l}\mathrm{H} \\
\mathrm{El}\end{array}$} & 0.0 & 0.0 \\
\hline $\mathrm{H}[\mathrm{end}]$ & 0.0 & 0.0 & & & & & 0.0 & 0.0 & & 0.0 & 0.0 \\
\hline
\end{tabular}

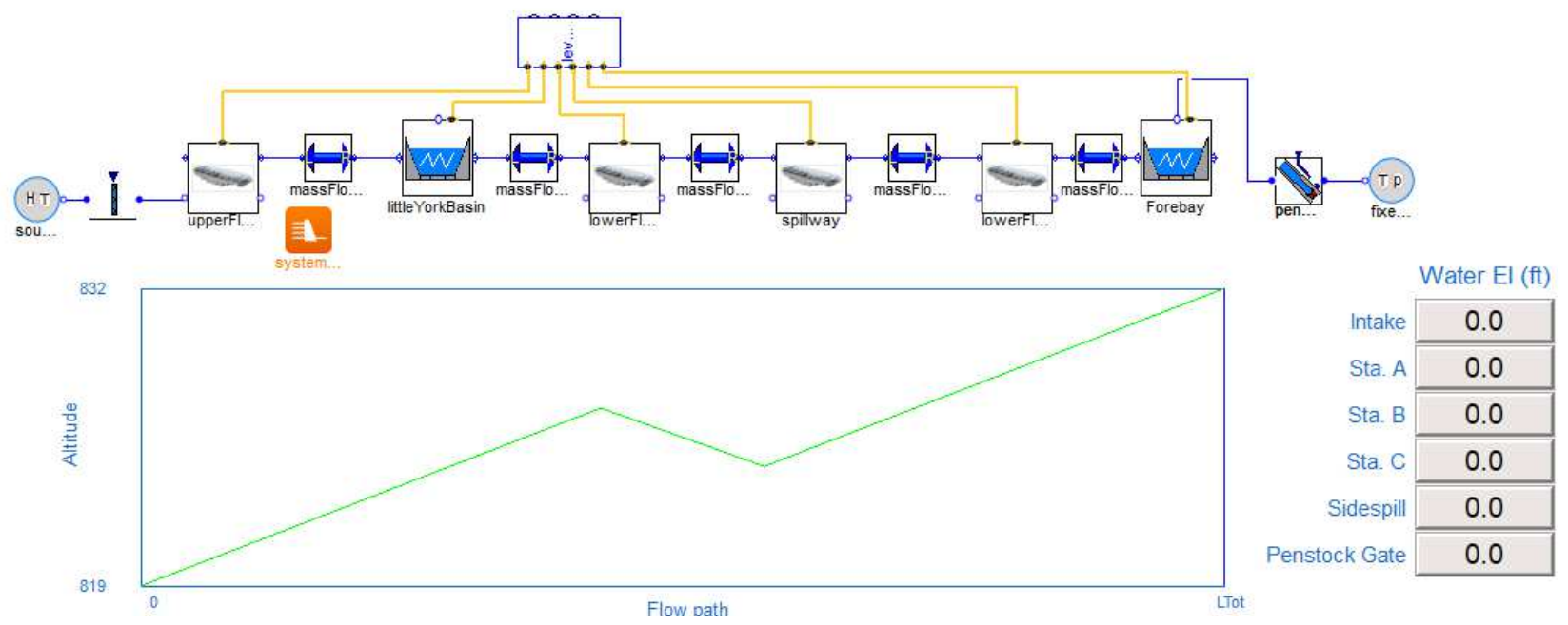

Figure 1. Flume system model including visualizers

Individual components are discussed in more detail in the following sections.

\subsection{Intake Gate}

The intake gate is modeled as a linear valve with the gate velocity as an input as shown in Figure 2. The valve velocity is integrated to provide a valve lift. The valve is characterized based on an estimate of the gate position at maximum flow.

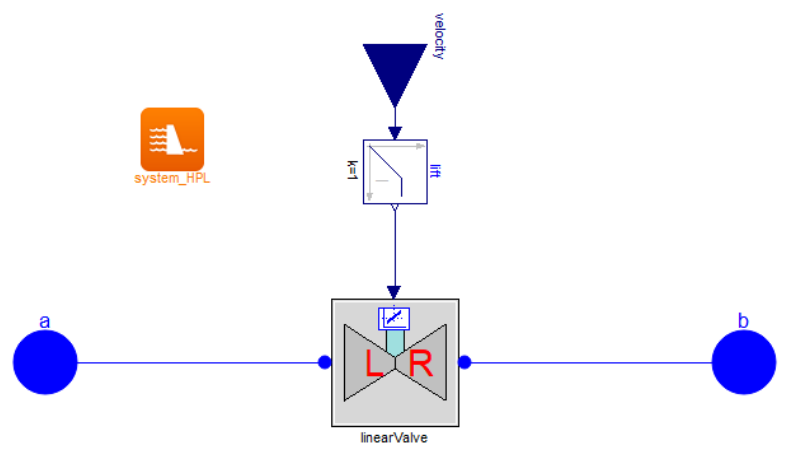

Figure 2. Intake gate model

\subsection{Flumes and Spillway}

The upper and lower flumes and spillway are modeled as reservoirs with varying elevation, width, cross section shape, and maximum height along the discretized length of the flume. The flumes are discretized models with combinations of volumes with open channel flow between adjacent volumes. The upper flume is rectangular shape and the lower flume is trapezoidal. The spillway is modeled as a separate component to allow increased resolution in the area that is most important for spill control. The upper flume is discretized into 80 segments while the lower flume and spillway include roughly 10 segments as they are significantly shorter.

Hydro Power Library provides models for friction in open and closed channel flow but allows flexibility for custom models to be implemented. For this work, a custom friction model was implemented and integrated into the reservoir model. The friction model is based on the standard Manning equation with the Kutter roughness coefficient (Sellin, 1970). The roughness coefficient for concrete ranges from 0.01 to 0.015 .

Since the focus of the controls development is avoidance of spill, it is critical that the flume system flows correctly at different depths. Unfortunately no detailed information was available prior to commissioning for the flow of the overall system. However, operator setpoint data was available for the upper flume depth as a function of flowrate. A model of the upper flume was used to calibrate the roughness coefficient for the friction model as shown in Figure 3. The results from the calibrated model are shown in Figure 4 and compared with the setpoint data. Given that no additional flow information was available, the same coefficient was then used for the lower flume and the spillway. 


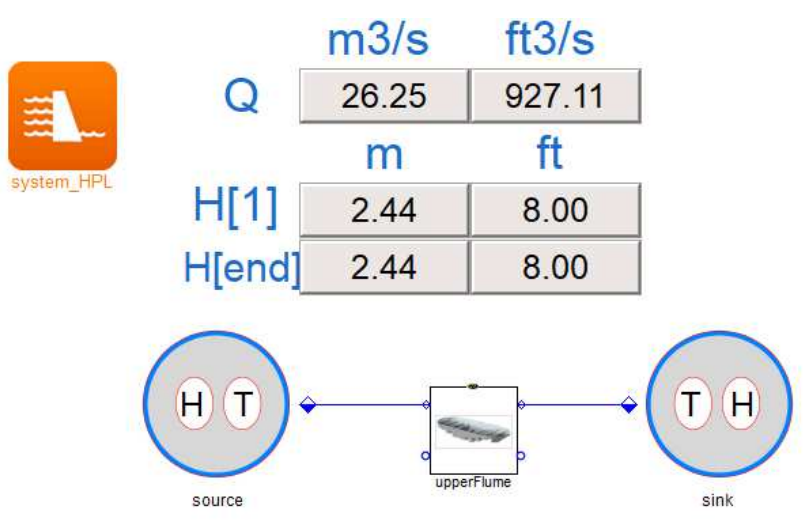

Figure 3. Calibration model for upper flume

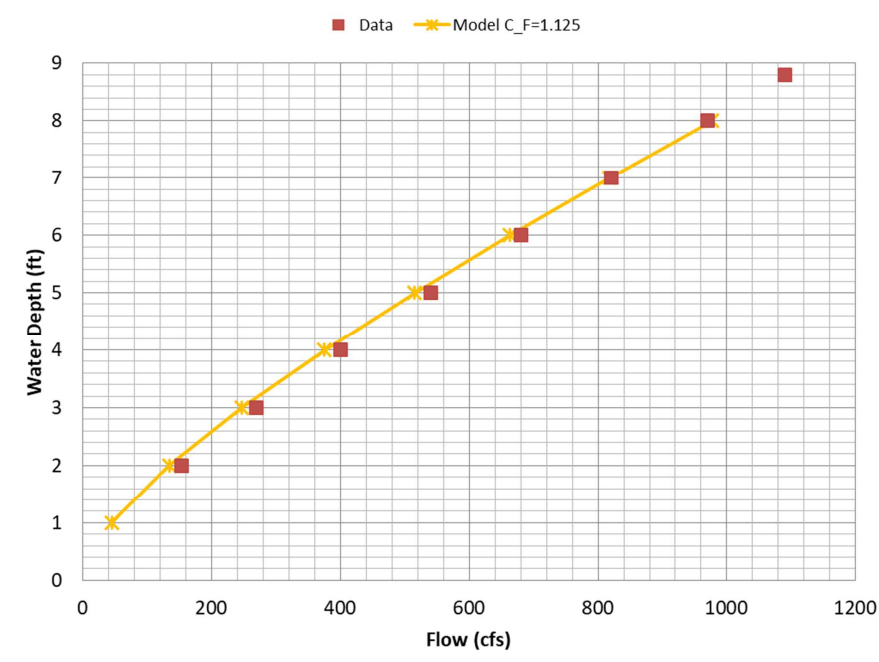

Figure 4. Calibration of upper flume friction based on operator setpoint data

\subsection{Reservoirs}

The reservoirs for the basin and forebay are based on the open volume component in Hydro Power Library. The open volume component contains conservation of mass and energy equations for a variable volume. The open volume component in the library allows geometry specification as shown in Figure 5. However, the reservoirs in the flume system do not map to a simple geometry specification as the reservoir geometry for the actual flume system is highly irregular due to topological variations of the geography. Thus, a custom component was created to allow a flexible specification of the depth and volume relationship as a table.

The data for the basin and forebay are shown in Figure 6. Using this table-based representation for the depth and volume relationship calculated from the actual reservoir geography, the model can accurately reflect the detailed, irregular geometry for reservoir capacity and depth without requiring a complicated geometric implementation.

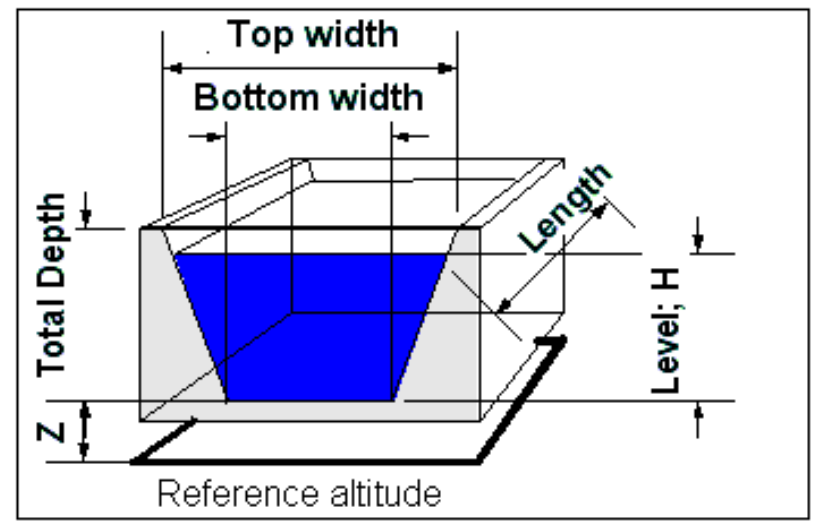

Figure 5. Base open volume component geometry specification in Hydro Power Library

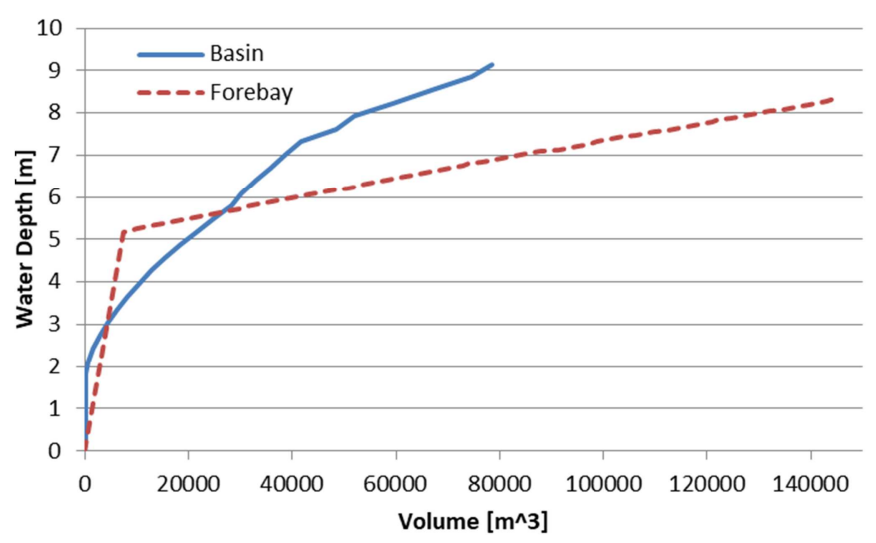

Figure 6. Reservoir depth-volume characterization

\subsection{Penstock with Valve}

The penstock that leads to the turbine inlet is connected to the forebay reservoir. The penstock valve is a linear valve that is characterized to deliver the maximum plant flow at a flow command of 1 . Figure 7 shows the model used to characterize the penstock valve. This model includes the forebay at a specified height and then the penstock with a specified downstream pressure at the outlet. Based on the location of the penstock connection to the forebay, the hydrostatic pressure drives the flow in the penstock. The results from running the valve at the maximum flow command are shown in the visualizers in Figure 7.

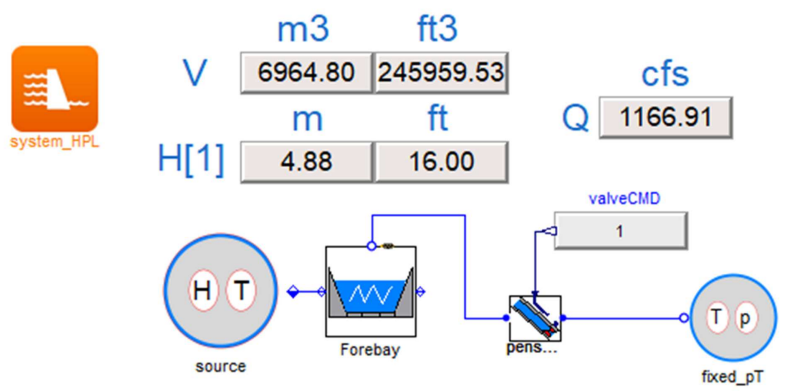

Figure 7. Test case for penstock valve characterization 


\subsection{Flume System Visualization}

When simulating the flume system, results for water depth, flowrate, pressures, etc. at any location in the flume system are readily available. Figure 8 shows typical results from a flow rate step test. The time for a flow disturbance to travel downstream in the flume system is clearly shown by the delays in the elevation response at different locations along the waterway.

While these local results are valuable, they can be difficult to interpret to get a good overall picture of the spatial distribution of the water in the flume system, including waves. Thus, a custom component was developed to aggregate the information from the individual flume system components and visualize the entire flume system using the diagram layer animation features in Dymola (Dassault Systemes, 2018).

Figure 9 shows an example of the visualization of the flume system for a large change in inlet flow. The flow change begins from a steady state condition around $\mathrm{t}=320 \mathrm{~min}$. The visualization shows the invert elevation of the system (blue), maximum height of the system (green), and dynamic water elevation (red). The effect of the flow change is clearly seen progressing down the flume system, including wave behavior at different parts of the flume. When animated in Dymola, the visualization provides an animation of the flume system as a function of time and is critical for understanding the dynamics of the system and assessing the water depths relative to the spill limits. The combination of the detailed traces at a specific location as shown in Figure 8 and the overall visualization of the entire flume system as shown in Figure 9 provide a more complete view of the waterway dynamics.
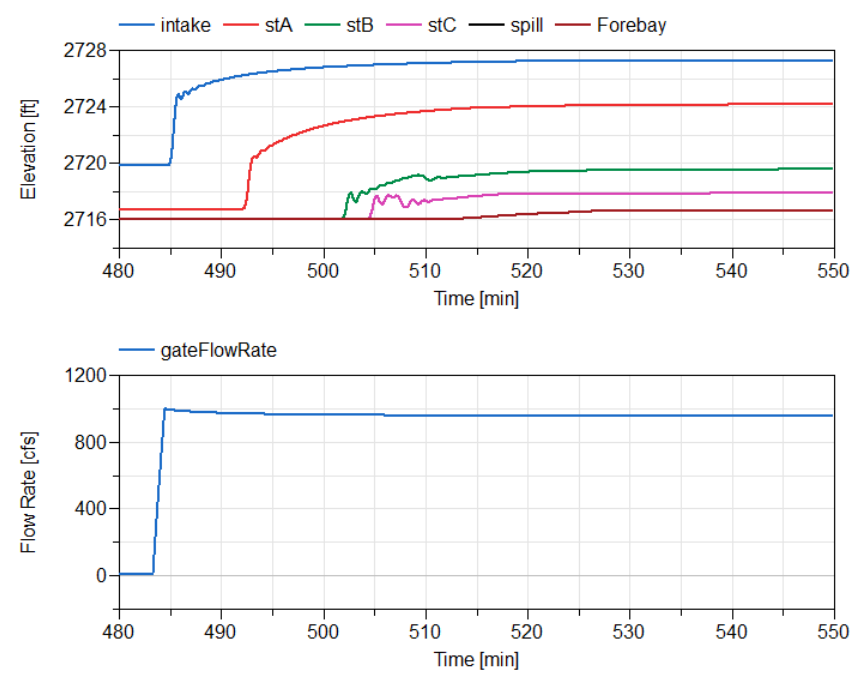

Figure 8. Results from flow rate step test

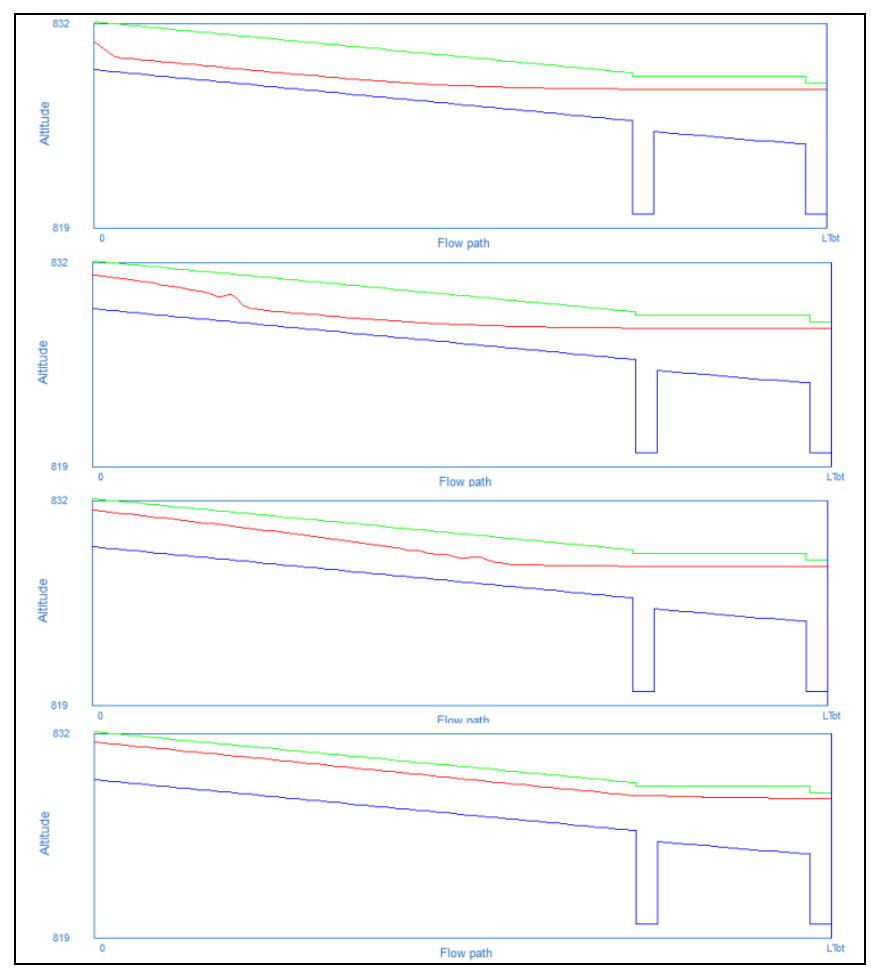

Figure 9. Flume system visualization for a large change in inlet flow at $\mathrm{t}=321 \mathrm{~min}, \mathrm{t}=326.67 \mathrm{~min}, \mathrm{t}=335 \mathrm{~min}$, and $\mathrm{t}=350 \mathrm{~min}$

\section{Controls Development and Integration}

For this plant system, there is concern for the environmental impact of any potential spill. Thus, the focus of the controls modernization is system control for spill. The control algorithm receives the forebay level as input and actuates the penstock valve. The actual discrete control algorithm is implemented in the model as in the hardware PLC logic and with the same calibration values. Due to confidentiality reasons, the actual control algorithm cannot be shown nor can the calibration values.

Figure 10 shows the aggregate penstock control block. This block includes several different control modes including open loop control, continuous control, and the actual discrete control implemented in the block controllerl. The reason for this controller structure is to allow different operating modes for the system including:

- Open loop for manual operation

- Continuous control for computational efficiency in establishing test conditions for the simulation

- Steady state detection

- Discrete control algorithm under development

Since the controller can operate in various modes, it is important that the transfer to the actual discrete controller occurs without disturbance to the penstock command. Thus, bumpless transfer is implemented as in the actual controller to ensure smooth transitions. 


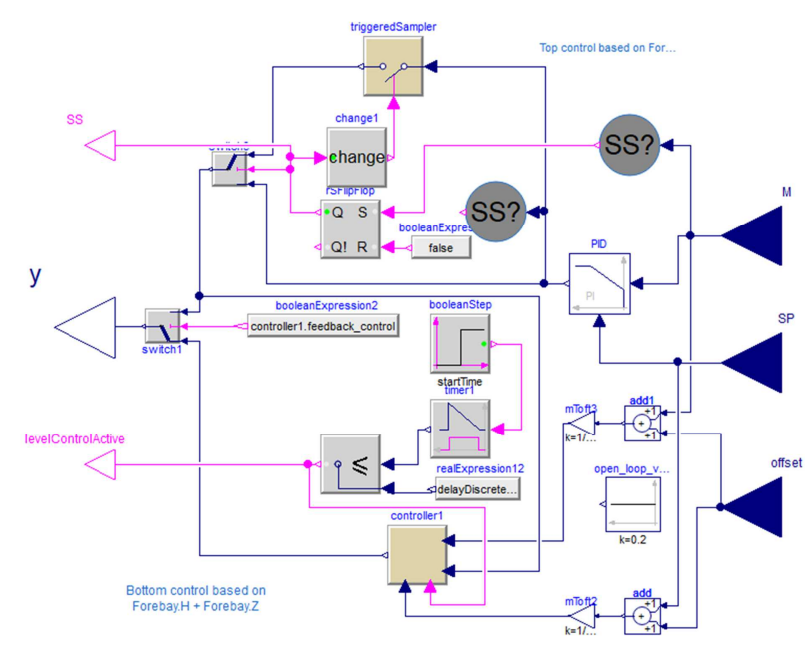

Figure 10. Penstock control including open loop, continuous control, and discrete control with switching

Figure 11 shows the model of the flume system with the integrated penstock control and gate control elements. This model is extended from the base flume system model in Figure 1 and simply adds the control elements. The gate control is simply for actuation of the intake system for testing purposes. The gate controller specifies the maximum gate opening velocity until the desired flowrate is achieved and then holds the gate position.

\section{Virtual Controls Verification}

The integrated model in Figure 11 serves as the test bench for the virtual controls development, calibration, and verification. This section describes and gives results from a virtual test plan that was conducted prior to the commissioning of the controls integration on the plant hardware. The virtual test plan was developed to prove out the physical model and calibrate the proprietary control algorithm. Though formal controls methods are certainly applicable to ensure controller stability, the calibration of the control algorithm was performed via execution of the virtual test plan with a focus on operating conditions that pose the most severe spillage risk and were also planned for execution in the plant commissioning.

\subsection{Flow Steps with Fixed Valve}

To test the overall system response, a series of flow step tests were conducted as follows:

- Run to steady state at initial flowrate and system level using continuous control for efficiency

- Initiate flow step but with fixed penstock command from initial flowrate (i.e. no controller)

\section{- Observe system response}

Figure 12 shows the system response to a flow step change but with relatively low flows. As the flowrate increases, the elevation increases since the valve command is fixed at the steady state value from the initial flowrate conditions. Figure 13 shows the system response to a flow step change but with a step from low to high flow. Under this condition, the system elevation increases rapidly because the valve command is fixed at the value for a much lower flow. The simulation is stopped due to the excessive level.

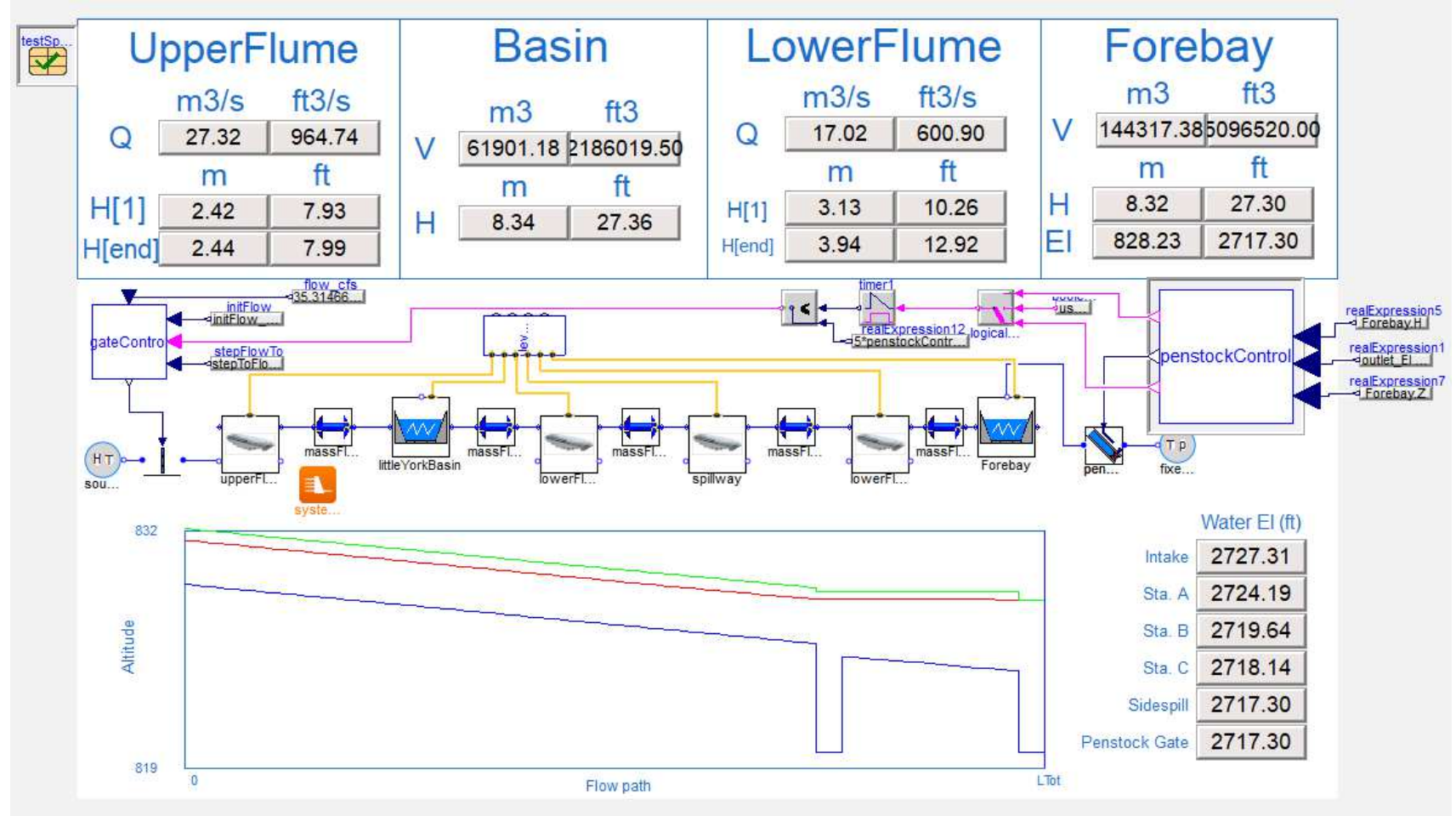

Figure 11. Flume system model with integrated penstock control and gate control 
For these tests and many of the subsequent tests, continuous level control is used to run the system to steady state at specified initial conditions. This control is simply to get the system to a desired initial state, and continuous control is used for computational efficiency. It should be noted that this control is completely separate from the actual discrete controls being prototyped and is only used to set the initial system state for testing purposes. Furthermore, no significant effort was spent calibrating the continuous control given its purpose to help initialize the system for dynamic testing. The continuous control can be used to simulate the system to a steady state and then hold the valve command to simulate manual control of the system.
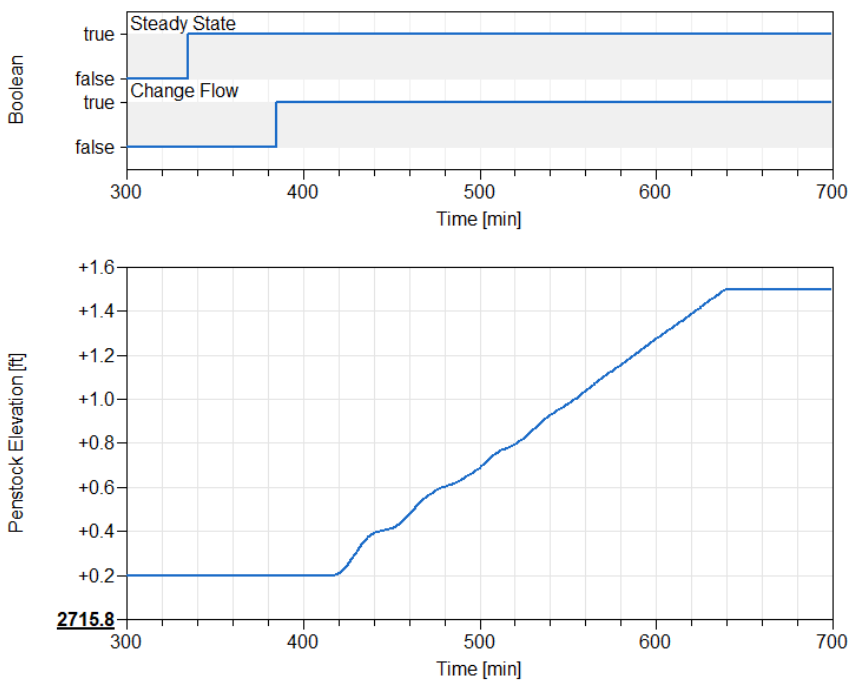

Figure 12. System response to flow step change, low flows
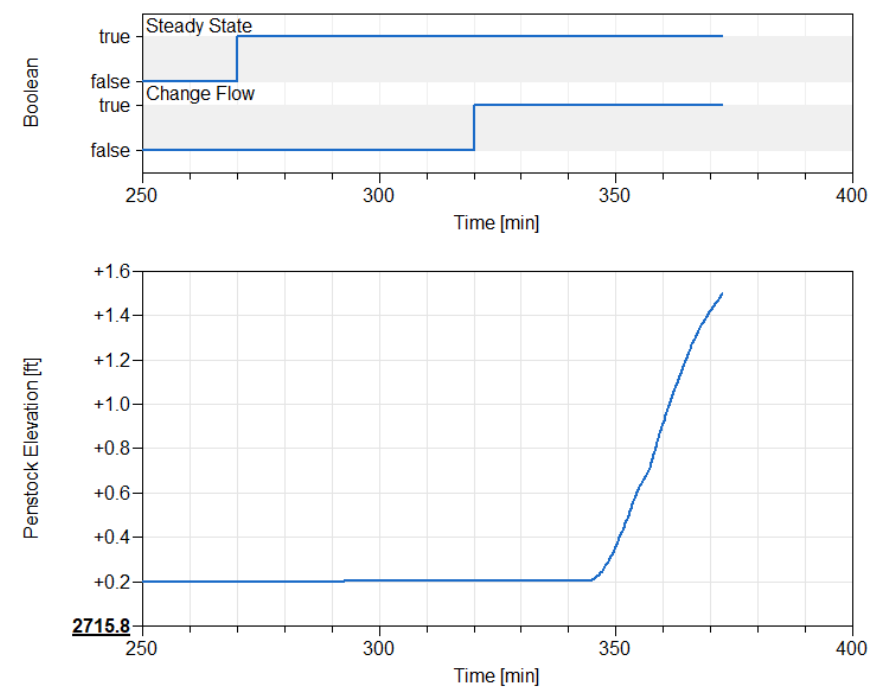

Figure 13. System response to flow step change, step to high flow

\subsection{Level Setpoint Steps}

A series of tests for controller stability were conducted as follows:

- At a specified flowrate, run to steady state at a specified system level using continuous control for efficiency

- Engage discrete controller and initiate a change in the system level setpoint

- Observe system and controller response

These tests were conducted at different flowrates and for both step up and step down in system level. Standard controller design metrics such as overshoot, undershoot, and settling time were used to calibrate the controller. Figure 14 shows results from a test with a step down in level control setpoint at both high and low flowrates. When the level setpoint change is initiated, the controller smoothly opens the penstock valve until the desired level is achieved and then ultimately returns to the initial opening since the flowrate is held constant in these tests. At the higher flowrate, the response is slower as the penstock command is saturated at maximum opening.
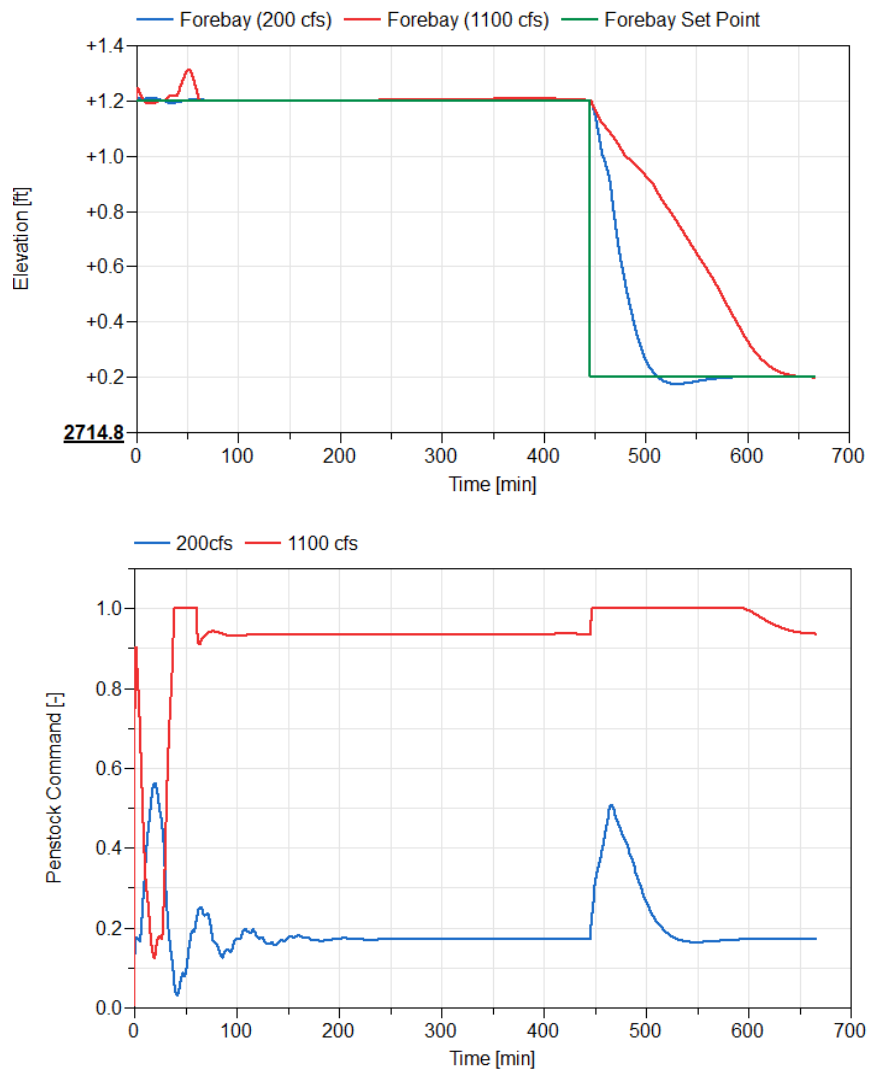

Figure 14. System response to level step down at low and high flows

Figure 15 shows results from a test with a step up in level control setpoint at low, medium, and high flowrates. When the level setpoint changes, the penstock valve closes to increase the system level. 
Once the desired level is achieved, the valve command opens again to allow the system to flow at the new system level. At the lowest flow command, the penstock valve actually closes completely before opening again.
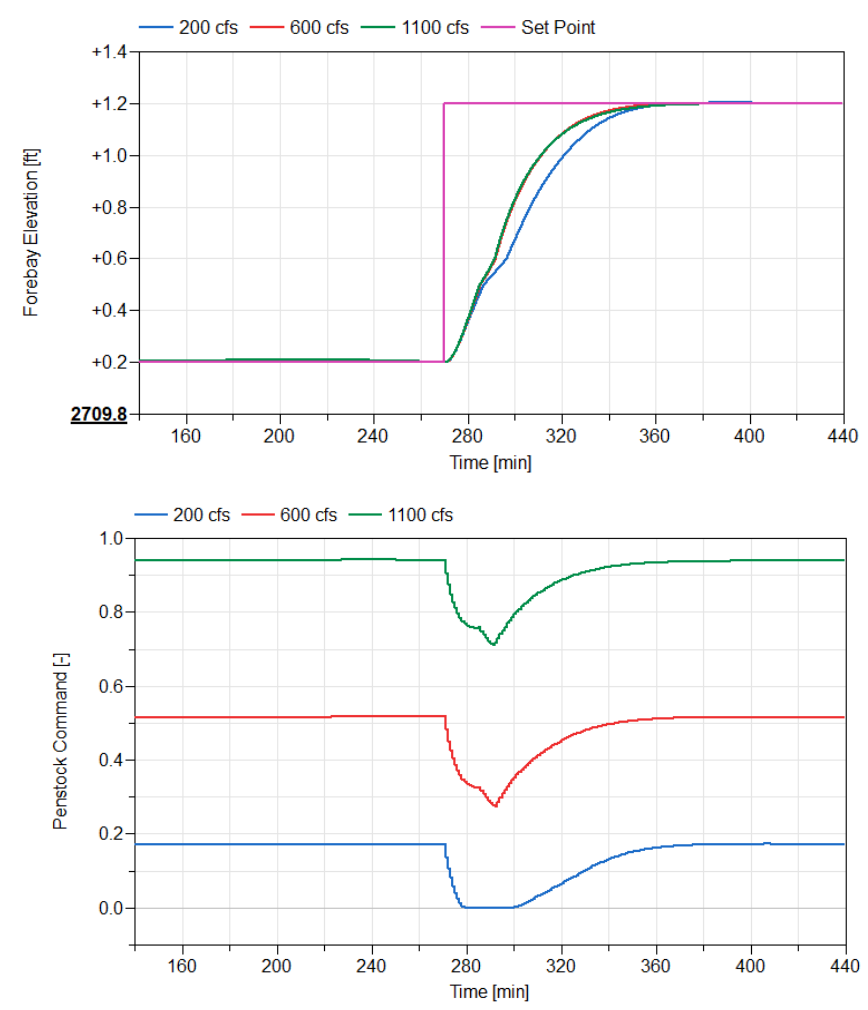

Figure 15. System response to level step up at low, medium, and high flows

\subsection{Oscillation Tests}

When there is a significant elevation difference between reservoirs, there is the potential for flow to oscillate between the reservoirs. A series of oscillation tests were performed to ensure that the controller did not cause the system to become unstable under this scenario. The tests were conducted as follows:

- At a high flowrate, run to steady state at a specified system level

- Switch off flow at intake to induce a level difference between reservoirs

- Engage active level control to observe interaction between active level control and level oscillations

Figure 16 shows results from the tests. When the flow at the flume intake turns off, the basin reservoir sees a drop in water elevation first. There is oscillating flow that exists between the two reservoirs as evidenced by the level oscillations. With active level control, the controller quickly closes the penstock valve to maintain the system level. The oscillating flow between the reservoirs eventually damps out, and there does not seem to be any adverse impact of the active level control on the oscillations. Though stability considerations are best evaluated via formal controls methods, these time domain test are useful for validating the controls performance.
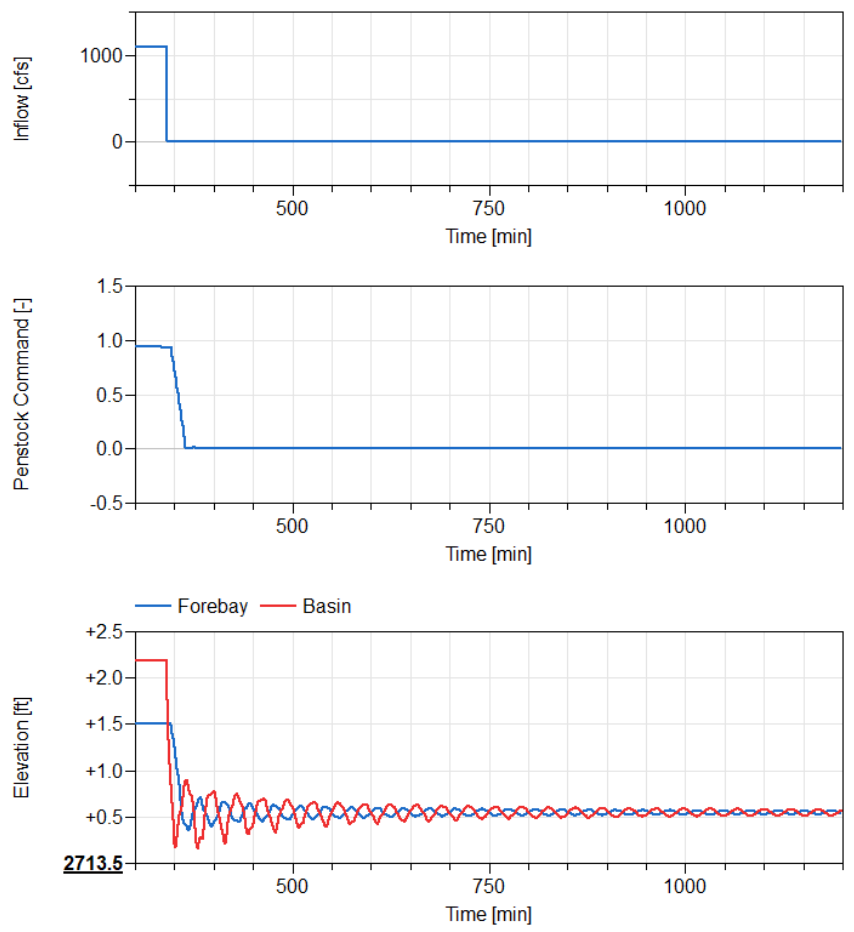

Figure 16. System response to oscillation test with active level control

\subsection{Max Flow Step Tests}

The highest risk of spilling occurs when the system experiences the maximum step from lowest flow to highest flow when the system level is high. To simulate this worst case condition, the following test was conducted:

- Run system to steady condition at very low flow and high system level with manual control

- Step to maximum inlet flow keeping manual control

- Observe controller and system response to flow increase

Figure 17 shows the response to the max flow step test. In this scenario, the controller as calibrated is able to arrest the system level and ensure that no spilling occurs. Figure 18 shows some sensitivity results to limits in the controller for the same flow step. With a low limit on the controller, the maximum elevation increases as expected.

These max flow tests were critical for the evaluation of the controller calibration and provided model-based verification of the controller under extreme conditions. As described in Section 5, plant commissioning conducted with the calibration developed from the virtual tests showed similar results. 


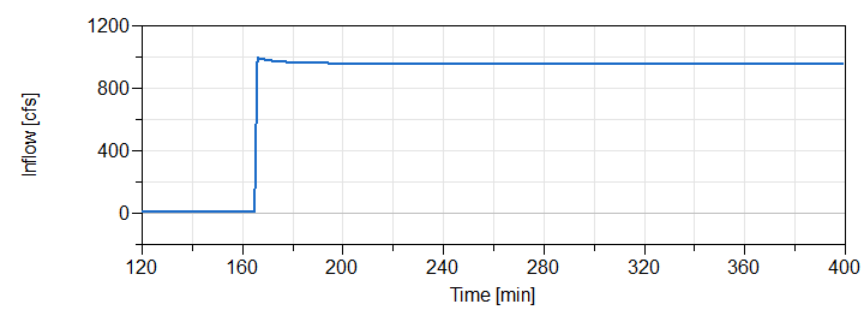

flow. Even with the penstock valve completely closed, it takes time to increase the system level due to the low intake flow. As expected, the system exhibits different time constants when subjected to flow steps up and down given the underlying volume dynamics required to change the system elevation.
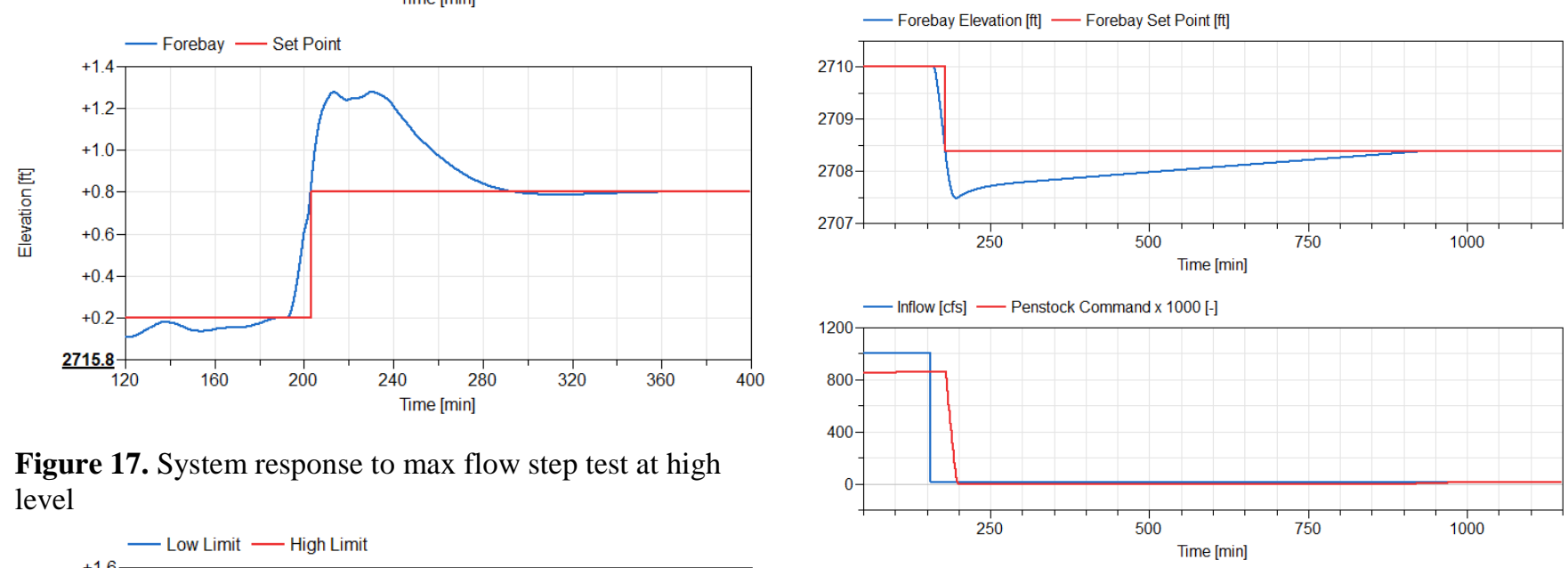

Figure 17. System response to max flow step test at high level

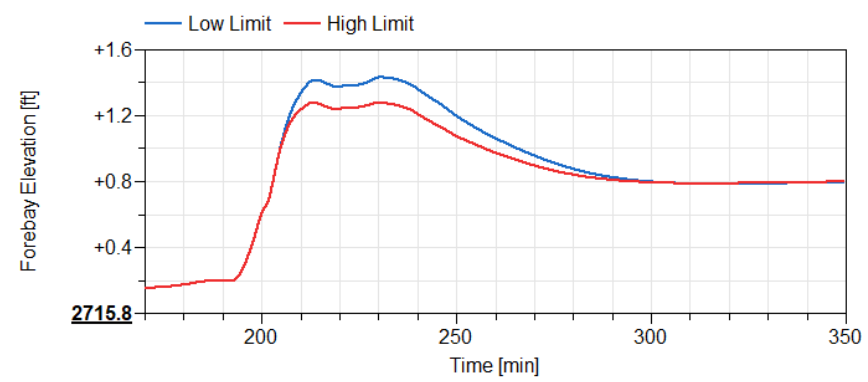

Figure 19. System response to min flow step test at low level

\section{Plant Commissioning}

After the model-based calibration and verification of the controller on the virtual test suite, the controller was commissioned on the plant. The controller as implemented in PLC logic for the plant level control is

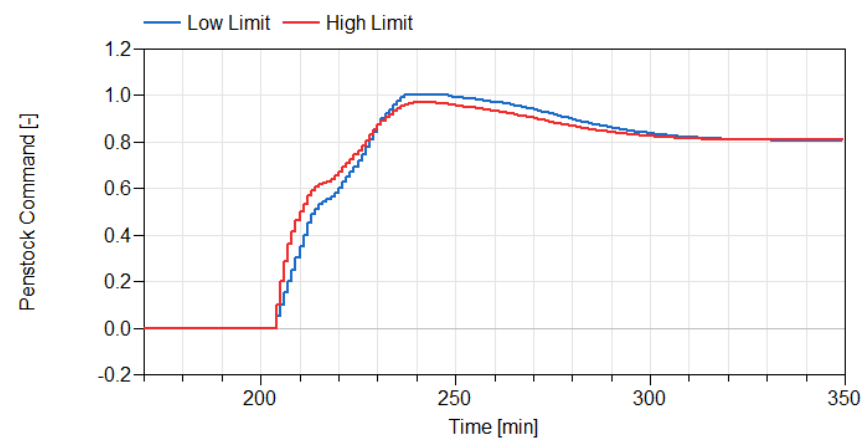

Figure 18. System response to max flow step test at high level, varying controller limits

Similar tests were also conducted at low system levels. The test scenario is as follows:

- Run system to steady condition at very high flow and low system level with manual control

- Step to very low inlet flow keeping manual control

- Observe controller and system response to flow decrease

Figure 19 shows results from this test. The controller is able to control the system level appropriately. Notice the long time constant required to increase the system level due to the very low inlet identical to that implemented in Modelica. A comprehensive test plan was executed as part of the commissioning to ensure signal integrity, controller response, actuator bandwidth and response, etc. over a wide range of operating conditions.

Selected results from the commissioning are shown below. The model-based calibration developed during the analytic work was successfully validated in the commissioning work. Only minor changes were required to handle some signal conditioning issues which were not anticipated and not simulated. Otherwise, the results predicted by the simulations were confirmed in the commissioning.

Figure 20 shows results from a step up test during the commissioning. The commissioning data is provided at 5 minute intervals. The intake setpoint is representative of the intake system flow. The system level response compares favorably to the similar test shown in Figure 17-Figure 18. The tests are not identical as they start at different initial levels. However, the response of the system level in terms of time constants and profile compare well. The calibration developed in the model proved capable of managing the spillage risk as commissioned on the plant. 


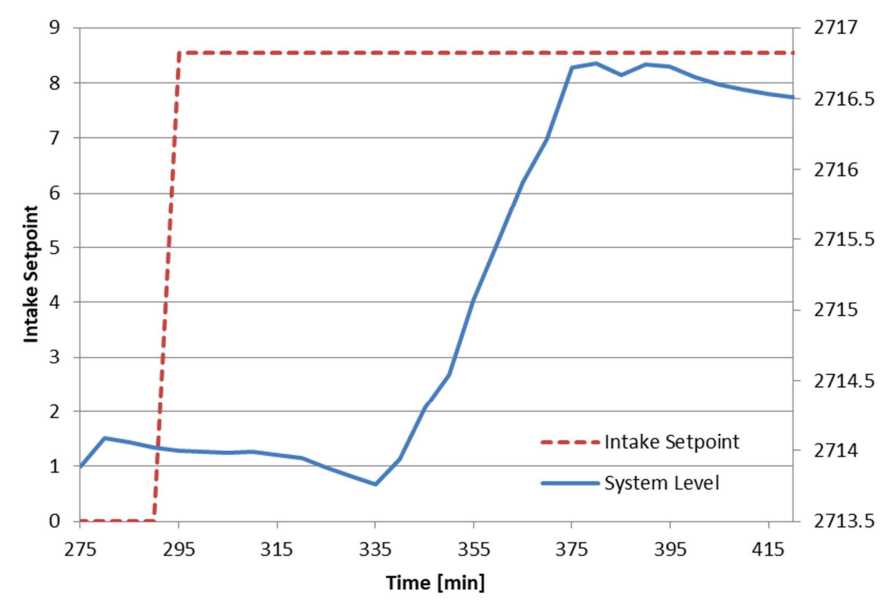

Figure 20. System response to max flow step test during commissioning

Figure 21 shows results from a step down test during the commissioning. Similar characteristics are seen when compared with the step down test in Figure 19 though the tests are not identical since the commissioning step down test starts from a high level while the simulations started from a low level. The simulations take much longer to respond due to the different starting level as the penstock command saturates.

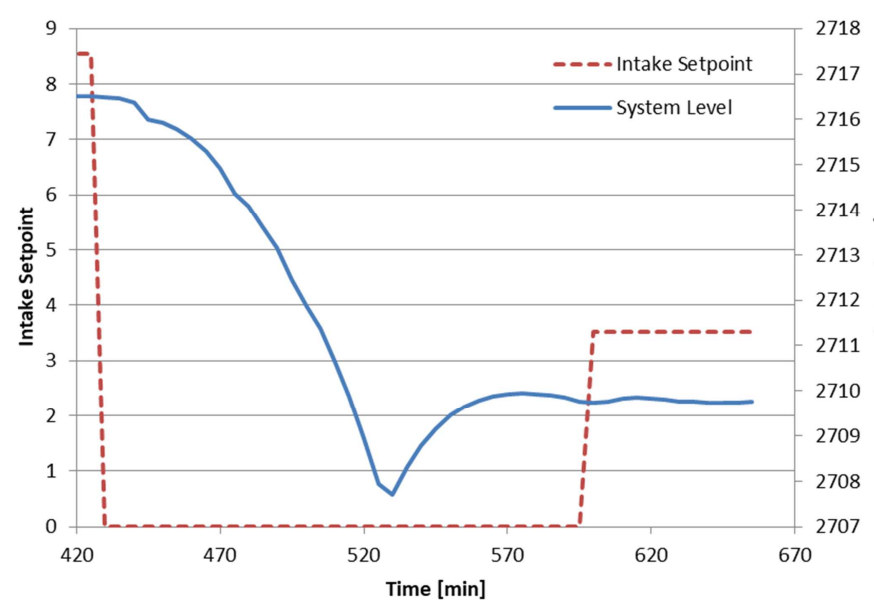

Figure 21. System response to min flow step test during commissioning

Based on the results from the commissioning, several different actions to improve the overall modelbased controls development process were identified:

- Any flow data available when building the model is critical as it allows verification of the system response early in the development process

- Simulating not just the overall system response but also any dynamics in the sensor system will provide better input signals for the model-based calibration and potentially reduce/eliminate onsite calibration work for a more robust virtual calibration process
- Steady state initialization of the system model would reduce the time spent waiting for the system to reach steady state and avoid the extra logic in the controller to control the test conditions

\section{Summary}

This paper describes the model-based control system development for a hydroelectric power plant to ensure water level control and mitigate spillage risk. The paper gives an overview of the work to develop a waterway system model with Hydro Power Library and associated controls. The control algorithm was prototyped in the model, and a model-based calibration process was used to verify the algorithm and calibration over a virtual test suite.

Following the analytic work, the controller was commissioned on the plant and successfully verified based on a set of commissioning tests. The algorithm prototyped in Modelica is identical to the PLC logic implementation used on the plant. Results from the model compare favorably to the commissioning tests with only minor changes required to the calibration due to unanticipated signal conditioning issues. Overall, the model-based approach proved capable of predicting the waterway dynamics and for model-based calibration of the control strategy. Future work could include additional calibration of the model based on the commissioning data and adding capability to the model to capture the sensor system dynamics and thus enable an even more robust analytic calibration process. Formal controls methods, including a linearized model, to ensure controller stability are also considered as potential future work.

\section{References}

Dassault Systemes, Velizy, France (2018) Dymola 2018.

https://www.3ds.com/productsservices/catia/products/dymola

Magnúsdóttir, A. and Winkler, D., "Modelling of a Hydro Power Station in an Island Operation", Proceedings of the $12^{\text {th }}$ International Modelica Conference, May 15-17, 2017. Prague, Czech Republic.

Modelon AB, Lund, Sweden. (2018). Hydro Power Library. http://www.modelon.com/products/modelon-librarysuite/hydro-power-library/

Sellin, R., Flow in Channels, Gordan and Breach Science Publishers, New York, 1970.

Winkler, D., Thoresen, H., Andreassen, I., Perera, M., and Sharefi, B., "Modelling and Optimisation of Deviation in Hydro Power Production", Proceedings of the $8^{\text {th }}$ International Modelica Conference, March 20-22, 2011. Dresden, Germany. 
